

\title{
Variation in centric relation in the conscious and the anesthetized patient
}

\author{
Carole JOLIVET
}

CECSMO Report supervised by Professor Robert GARCIA

Every practitioner undertaking a case of dental rehabilitation has as one of the major goals of successful treatment making the patients' centric occlusions coincide with their occlusion of maximum inter-cuspation in order to assure that their masticatory apparatus will function harmoniously.

Specialists in dentofacial orthopedics, while always aiming at improving facial esthetics, enshrine the re-establishment of good masticatory functioning as an essential goal and to do this they must respect the fundamental principles of occlusion.

Some serious skeletal deformities that cannot be completely resolved by dentofacial orthopedics alone require surgical intervention. These orthognathic procedures should be designed to achieve the same occlusal objectives of establishing skeletal and facial equilibrium with dentitions in normal Class I Angle occlusion and the condylar heads seated correctly in the mandibular fossas. This means that the orthodontist dealing with conscious patients and maxillo-facial surgeons operating on the same individuals when they are anesthetized will seek the same mandibulo-cranial relationship, the reference articulation, or centric relation.

Because general anesthesia causes complete neural relaxation of the muscles that are an important element controlling centric relation surgeons need to know if it modifies this articular reference position. If it does, surgeons then have to know what, if any, adjustments they should make in response.

In order to answer these questions we established an experimental protocol to compare the centric relation of the conscious patient $(\mathrm{CR})$ to the centric relation of the same patient when anesthetized (CR 2). 


\section{1 - EXPERIMENTAL PROTOCOL}

The objective of this study is to respond to these questions:

- is there a difference between the CR of the conscious patient and the CR of the same patient when anesthetized?

- if the first question is answered in the affirmative are the sex and the facial type of patients factors that favor or are predictive of this difference?

\section{1 - 1 - Analysis of the sample}

The sample consisted of thirty-two patients, with an average age of 23 years, 9 months, 21 female $(66 \%)$ and 11 male (34\%). The youngest patient was $15 \frac{1}{2}$ years old and the oldest was 40 years and 4 months old. Twenty-five per-cent of the patients were classified as skeletal Class III and $75 \%$ were skeletal Class II. The distribution of the patients according to facial typology in the vertical sense was $9 \%$ normodivergent, $47 \%$ hyper-divergent, and $44 \%$ hypo-divergent. We excluded all subjects with malfunction of one or both of the temporo-mandibular joints.

\section{1 - 2 - Protocol}

\section{1 - 2 - 1 - Registering centric relation in the conscious patient}

We followed Lauritzen's technique in taking impressions of the patients, located the hinge axis with an Almore arch, and registered centric relation in the single appointment when surgical arches were placed. Using Dawson's two-handed manipulation technique $6,7,8$ we registered centric three times (to evaluate its reproducibility) with the aid of two laterally placed morsels of polyvinyl siloxane (Rigadur ${ }^{\circledR}$ ).

\section{1-2-2-Mounting on the articulator (DENAR)}

For the maxillary impression we used a double base, Lauritzen, 1964, Split-Cast method of articulator adjustment technique to verify the reproducibility of centric relation. Then we mounted the upper and lower models on the Denar Mark $\|{ }^{\circledR}$ articulator.

\section{1 - 2 - 3 - Registering centric relation on the anesthetized patient}

We carried out this second procedure in the operating room taking two registrations, CR 2 and CR 2', using Dawson's two-handed technique just after naso-tracheal intubation and before the operating field was set up, with the same material employed for conscious patients, Rigadur ${ }^{\circledR}$. The anesthetist then gave the patient, who was then at the end of the induction stage, a muscle relaxant: Utiva ${ }^{\circledR}$ (remifentanyl, central analgesic), Propofol $^{\circledR}$ (hypnotic), Tracrium $^{\circledR}$ (atracurium besilate) Midazolam $^{\circledR}$ (benzodiazepine) in association.

\section{$1-2-4$ - Measuring the variations between the $C R$ and the $C R 2$ registrations under general anesthesia}

We measured the variations between registrations of centric relation 




with the Denar Slidematic ${ }^{\circledR}$ that reveals condylar positions of CR (1), (2), and (3) on a millimeter sagittal and
Figure 1

Orthonormal landmarks, after Laplanche ${ }^{4}$. frontal plane with the help of a gauge (fig. 1).

\section{2 - RESULTS}

\section{2 - 1 - Dispersion of the results sagittally}

One can superimpose the emergence points, centered on CR 1 of the conscious patient to observe the distribution of the points CR 2 and CR 2' of the anesthetized patient (fig. 2).

\section{2 - 2 - Statistical study of the results}

\section{$2-2-1$ - Middle point of the RC 2 and the statistical analysis}

We calculated for each registration of each condyle the average CR 2 .
- Right condyle (table 1)

- Left condyle (table 2)

We made our statistical analysis with a small sample Student $t$-test that compares the average to 0 for each coordinate. We found a significant difference for the two registrations between the right condyle and the left condyle.

\section{2-2 - 2- Influence of parameters on the position of CR 2}

We tested a number of models for each registration for each condyle.

First, we used the multivariate analysis of variance, Manova, to test the influence of each parameter, sex, type, and class, on the ensemble of the three coordinates. In no case did 


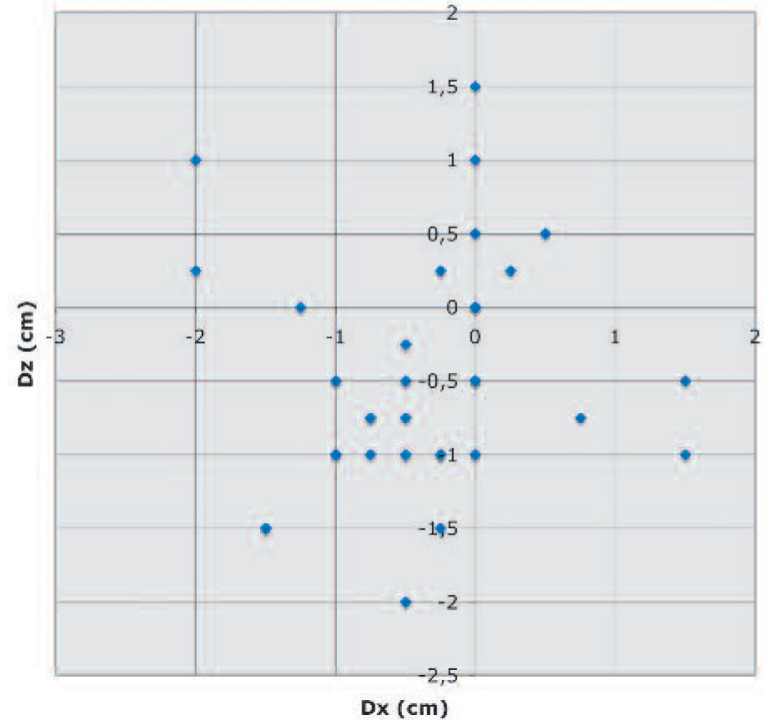

Figure 2

Distribution of the hinge axis under anesthesia with respect to the hinge axis of the conscious patient on the sagittal plane.

\section{we find any influence from any of these three parameters.}

Next, to refine the results, the influence of each parameter on a coordinate was sought using a model analysis of variance, Manova. In this way it was shown that the variable class has a significant effect on the $Z$ coordinate with respect to the right condyle in registration 1 and that the variable type had a significant effect on the $\mathrm{Z}$ coordinate in registration 2 , on both condyles.

\begin{tabular}{|c|c|c|c|c|c|c|}
\hline & \multicolumn{3}{|c|}{ Registration 1} & \multicolumn{3}{|c|}{ Registration 1} \\
\hline  & $\begin{array}{l}\text { Coordinate } \\
\qquad \mathrm{dx}\end{array}$ & $\begin{array}{l}\text { Coordinate } \\
\text { dy }\end{array}$ & $\begin{array}{l}\text { Coordinate } \\
\qquad d z\end{array}$ & $\begin{array}{l}\text { Coordinate } \\
\qquad \mathrm{dx}\end{array}$ & $\begin{array}{l}\text { Coordinate } \\
\text { dy }\end{array}$ & $\begin{array}{l}\text { Coordinate } \\
\qquad d z\end{array}$ \\
\hline $\mathrm{N}$ & 32 & 32 & 32 & 32 & 32 & 32 \\
\hline Mean & -0.30 & 0.08 & -0.21 & -0.38 & 0.05 & 0.48 \\
\hline Std & 0.57 & 0.31 & 0.75 & 0.55 & 0.35 & 0.60 \\
\hline Median & 0.00 & 0.00 & 0.00 & -0.25 & 0.00 & -0.50 \\
\hline Min. & -1.50 & -1.00 & -2.00 & -1.50 & -1.00 & -2.00 \\
\hline Max. & 1.00 & 0.60 & 1.50 & 1.00 & 0.70 & 1.00 \\
\hline
\end{tabular}

Table 1

Right condyle. 


\begin{tabular}{|c|c|c|c|c|c|c|}
\hline & \multicolumn{3}{|c|}{ Registration 1} & \multicolumn{3}{|c|}{ Registration 1} \\
\hline 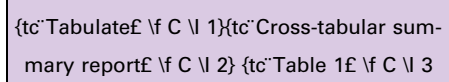 & $\begin{array}{l}\text { Coordinate } \\
\qquad \mathrm{dx}\end{array}$ & $\begin{array}{l}\text { Coordinate } \\
\text { dy }\end{array}$ & $\begin{array}{l}\text { Coordinate } \\
\quad d z\end{array}$ & $\begin{array}{l}\text { Coordinate } \\
\qquad \mathrm{dx}\end{array}$ & $\begin{array}{l}\text { Coordinate } \\
\text { dy }\end{array}$ & $\begin{array}{l}\text { Coordinate } \\
\quad d z\end{array}$ \\
\hline $\mathrm{N}$ & 32 & 32 & 32 & 32 & 32 & 32 \\
\hline Mean & -0.27 & 0.08 & -0.27 & -0.27 & 0.05 & -0.48 \\
\hline Std & 0.65 & 0.31 & 0.65 & 0.62 & 0.35 & 0.61 \\
\hline Median & 0.00 & 0.00 & 0.00 & 0.00 & 0.00 & -0.50 \\
\hline Min. & -2.00 & -1.00 & -1.50 & -2.00 & -1.00 & -2.00 \\
\hline Max. & 1.50 & 0.60 & 1.50 & 1.50 & 0.70 & 1.00 \\
\hline
\end{tabular}

Table 2

Left condyle.

The statistical analysis disclosed that there was a significant difference, at the $5 \%$ confidence level, between CR of the conscious patient and CR 2 under general anesthesia for the two registrations made under general anesthesia.

The average condylar displacement under general anesthesia, in the sagittal plane is postero-inferior:

- Average $D x=-0.3 \mathrm{~mm}$.

- Average Dz $=-0.4 \mathrm{~mm}$.

- Average Dy $=0.06 \mathrm{~mm}$ toward the right.

The Manova analysis showed that sex, facial typology, and skeletal class had no influence on the determination of CR 2 under general anesthesia.

\section{$3-$ DISCUSSION}

\section{3 - 1 - Discussion of results}

It is important to remember that under general anesthesia, the determination of condylar reference position depends only on certain factors:

- the biometric relationship of the temporal eminence to the condylar disc;
- the ligamentary and muscular viscoelasticity of each of these two tissues;

- gravity;

- the surgeon's preliminary manipulation.

According to our results only $\mathbf{1 8 . 8 \%}$ of condylar reference positions were identical before and during general 
anesthesia. Patients under general anesthesia were able to exert very little influence on this reference position. Twelve and a half $\%$ have a position close to, but not truly identical to $C R$, either because of a transverse deviation or a lack of co-incidence between the two condyles.

For the $\mathbf{3 8 . 3} \%$ of the positions more postero-inferior and the $\mathbf{3 . 2} \%$ of the positions more posterior, the ligamentary and muscular visco-elasticity as well as the operator's two-handed manipulation, identical to that used on conscious patients, were not sufficiently strong enough overcome the forces of gravity acting on different muscular, ligamentary, discal, and osseous structures. This tends to reinforce the general hypothesis that without tonic muscular action and neuro-sensory activity, the determination of CR under general anesthesia can never be identical to its determination on conscious patients.

How can we explain the $\mathbf{5 . 5 \%}$ of positions that were more posterosuperior, that is higher and more retruded than conscious CR? The most rational explanation would be that an over-zealous manipulation had caused a distal luxation caused by the general anesthesia's depriving the patient of normal reflex protection and the operator of clinical guidance signs.

A partial luxation of the disc having taken place unnoticed clinically can explain the $\mathbf{3 . 1 \%}$ of more superior positions and the $\mathbf{6 . 2 \%}$ of more anterior positions (both antero-superior and simply anterior). The total luxation of the disc would appear under general anesthesia.
An error in manipulation would seem to be the most logical explanation for the $\mathbf{2 . 3} \%$ of the more anteroposterior positions. The registration of mandibular-cranial relationships of patients with serious skeletal deformities is especially delicate because no wax bite or morsel bit technique is adaptable enough for them. Add to this the problems associated with general anesthesia, especially the effect on the tongue that is pushed forward between the dental arches by the intubation. This, then, is certainly the principal cause of the dispersion of our results.

However, our results showed the same tendencies as those obtained in previous studies by Posselt ${ }^{13}$ and Boucher and Jacoby ${ }^{4}$, McMillen $^{12}$, Zak et al. ${ }^{15}$, and Bamber et al. ${ }^{2}$, which all showed that under general anesthesia the condyle has a strong propensity to situate itself in a lower and more backward position.

\section{3 - 2 - Discussion of results in relation to sex and facial typology}

\section{3-2 - 1 - According to sex}

Ligamentary and muscular viscoelasticity always persisted during the determination of CR under general anesthesia. In hyper-relaxed anesthetized subjects, manipulation that is only slightly more forcible than normal could generate fairly large differences in condylar position.

Given that estrogens make women more susceptible to hyper-relaxation 
than men, it would be reasonable to anticipate that general anesthesia would generate greater condylar differences in women than it does in men. But although we found that the manipulation generated particularly large condylar differences in one anesthetized and hyper-relaxed female patient, the results our sample provided did not show any significant differences between men and women.

\section{3-2 - 2-According to facial typology in the vertical sense}

The shape of the head of the condyle with respect to the mandibular fossa seems to have some importance. The natural blocking effect varies between individuals. The presence of a slim condyle in a rather flat fossa, in hyper-divergent individuals, seems to make determination of a reproducible reference position more difficult to obtain. On the other hand, a larger condylar head in a well-defined fossa, in individuals tending toward hypo-divergence a certain natural blocking action exists. The results of our work, classified according to facial typology, showed no differences between hyper-divergent and hypo-divergent subjects, based on the Manova multivariance analysis. In terms of positions, CR of hyper-divergent subjects was located on the average more postero-inferiorly than it was with hypo-divergent patients in the sagittal plane and, in the transverse plane, more to the right.

\section{3-2 - 3- According to facial typology in the sagittal sense}

There is no significant difference between subjects with Class II and those with Class III skeletal occlusions, according to the multivariate analysis. In terms of positions, Class III subjects were displaced on average more postero-inferiorly than Class II subjects.

\section{$3-3$ - Discussion of results in a surgical context}

We must, however, situate our protocol in an orthodontic-surgical context. The occlusal objectives of surgeons being the same as those of orthodontists, surgical success is defined by the achievement of a post-surgical centric occlusion intercuspation relationship. Accordingly, in the course of the intervention surgeons seek to fix different skeletal elements in those positions that will best encourage the desired articular reference status of the centric relation of the patient when conscious.

As the intervention proceeds the position of the condyle in the mandibular fossa can be altered:

- by the difference between the two states: conscious and under general anesthesia,

- by the surgeon's manipulation,

- during the placement of the osteosynthesis material ${ }^{9}$.

Accordingly, the precise positioning of the condyle in the fossa prefigures the result of the intervention and, by the same token, global treatment. 


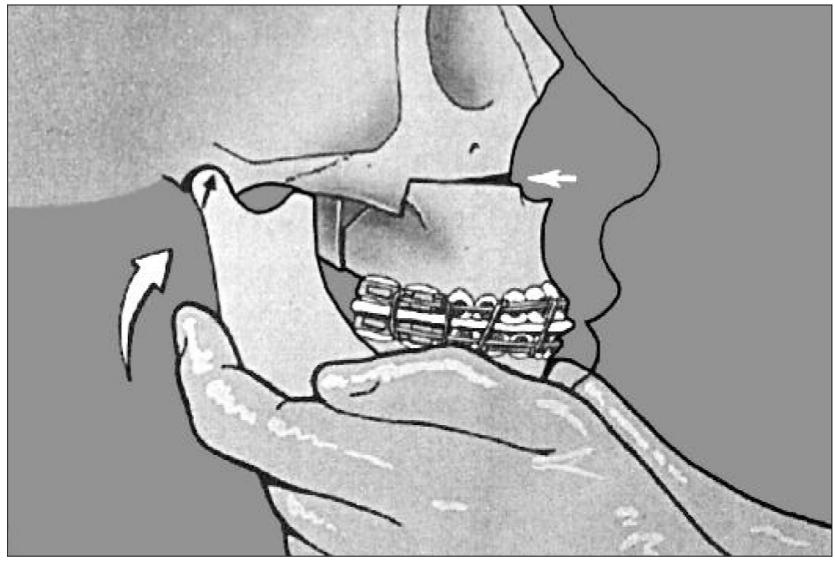

Figure 3

Operative manipulation during a Le Fort 1 procedure. After Bell .

\section{3-3-1-Method of positioning the condyle and fixation of the osteo- synthesis material}

\section{- Le Fort 1 type maxillary osteotomy}

According to the surgeon G. William Arnett ${ }^{1}$ operators can obtain the most physiological condylar position by bilateral positioning of the condyles. The surgeon moves them forward and upward thus avoiding compressing posteriorly in a manoeuver that is more or less identical to Dawson's two-handed manipulation. As with the conscious patient and no matter what technique is employed, the operator's sensitivity and experience are critical elements ${ }^{3}$ (fig. 3).

\section{- Sagittal type mandibular osteot-} omy (Obwegeser 1955 modified by Dalpont in 1959)

Merten and Halling, Benoist, and Brocard, among others, have described a variety of methods for repositioning the condyles. With the



Figure 4

Bilateral positioning of the condyles.

aid of bone clamps that grasp the proximal mandibular ramus surgeons position the condyle in the fossa by moving it upward and backward, seeking to slide it along the temporal tubercle until it reaches the base of the mandibular fossa ${ }^{1}$ (fig. 4).

The success of this manoeuver will depend on:

- the direction of the force applied to the condyle,

- the intensity of this force,

- the skill and the experience of the surgeon.

\section{3-3-2 - The fixation material}

Within the framework of sagittal mandibular or bi-maxillary osteotomies the fixation material applied to immobilize the condylar segment to the dental segment can influence condylar position in the three dimensions of space. Today surgeons use rigid interior fixation that reduces the time required for external bimaxillary fixation thus allowing patient re-edu- 
cation to begin more promptly than heretofore. The fixating plate is modeled to allow it to maintain passive contact with mandibular surfaces so that when screws are tightened condylar position is not altered ${ }^{9}$.

\section{3-3-3-Conclusion of the surgical context}

The condyles can, as we have seen, occupy different positions in the mandibular fossa, depending on how surgeons execute their placement. There is as yet no scientific consensus for correct condylar positioning during orthognathic surgery ${ }^{5}$. So it is possible, therefore, for surgeons to cause condylar compression, posterior, medial, or lateral, by altering the preoperative condylar position. Such surgical compressions, as well as the individual capacity of patients to adapt to the compression, varying in accordance with age, presence of systemic maladies, internal derangements, and noxious habits, can cause condylar resorption leading to long term relapse.

Moreover, if the condyles are in a position too low or antero-inferior in the mandibular fossas during the 8-week post-operative period, they will return to their initial pre-operative positions causing a rapid skeletal relapse ${ }^{1}$.

On the other hand, even if surgeons have performed the best possible manipulation under the most favorable

\section{4 - CONCLUSION}

Centric relation, which constitutes the condylo-cranial reference relation- conditions, on patients with a Class II skeletal pattern and a hypo-divergent type facial schema, condylar variations that are related to the general anesthesia may appear when patients awaken.

The surgeon locks the occlusion post-operatively by tying mandible and maxilla together in this condylar position of average postero-inferior relationship. As patients emerge from the anesthesia, the new occlusion engenders an articular constraint in this new postero-inferior position, which is maintained during the period of osseous consolidation. Phenomena of ligamentary distension may arise that could cause discal luxations ${ }^{10,14}$. This is why some patients may experience irreducible post-surgical luxations despite surgeons having performed the manipulation without exerting undue force.

If neuro-muscular physiological pressure forces the condyles back to their pre-surgical positions, the body of the mandible will, of course, also move forward.

A variety of possible outcomes may result. If incisal over-bite is substantial, the upper anterior teeth may be traumatized. Then, as their mobility persists, diastemas may appear. If the over-bite is not significant, the patient's occlusal relationship may assume a Class III tendency with a decrease in the residual post-surgical Class II posture or even pass into a frank Class III status.

ship in conscious patients, is an extremely precise recurring position 
over time. The anatomic and physiological integrity of different determinants of this position guarantee its reproducibility.

But the complete neuro-muscular relaxation that follows administration of general anesthesia compromises the necessary physiological articular equilibrium. This explains the results we obtained at the conclusion of our experimental protocol that investigated whether there is a significant difference between the condylo-cranial positions found in the conscious patient and those obtaining in anesthetized patients to whom operators had applied the same manipulations. In the anteroposterior sense, condylar displacement of the anesthetized patient is significantly more postero-inferior in centric relation than it is in conscious patients.

As a corollary observation, we found that sex and facial types in the sagittal and vertical senses could not be used as predictors for the extent of condylar displacement under general anesthe- sia as measured by the Manova multivariant model. Still, it should be noted that on average in the sagittal plane there is a greater postero-inferior displacement in subjects with Class III skeletal status, with ANB $<$ or $=0^{\circ}$ in a hyper-divergent facial type frame of FMA $<22^{\circ}$.

This observed variation of condylar position between the conscious patient and the same patient under general anesthesia can influence surgical results even though we were unable to quantify them beforehand because of variations in vertical dimension.

But an important question is can surgeons compensate for these variations as they manipulate the condyles while affixing the osteosynthesis material? Only their experience and their clinical sense can guarantee optimal condylar positioning. Orthodontic finishing, an indispensible post-surgical procedure, can help to assure, even perfect, the eventual result.

\section{REFERENCES}

1. Arnett GW. Entretien avec le chirurgien maxillo-facial G. William Arnett. Orthod Fr 1998;69:211-24.

2. Bamber MA, Abang Z, Ng WF, Harris M, Linney A. The effect of posture and anesthesia on the occlusal relationship in orthognatic surgery. J Oral Maxillofac Surg 1999;57:1164-72.

3. Bell WH, Mannai C. Entretien avec les Dr W. H. Bell et C. Mannai. Orthod Fr 1998;69:225-32.

4. Boucher L, Jacoby J. Posterior border movements of the Human mandible. J Prosthet Dent 1961; 11:836-41.

5. Costa F, Robiony M, Toro C, Sembronio S, Polini F, Politi M. Condylar positioning devices for orthognatic surgery: a literature review. Oral Surg Oral Pathol Oral Radiol Endod 2008 Aug;106(2):179-90.

6. Dawson PE. Position optimale du condyle de I'ATM en pratique clinique. Rev Int Paro Dent Rest 1985;5:11-31.

7. Dawson PE. Les problèmes de l'occlusion : évaluation, diagnostic et traitement. Paris : CdP, 1992. 
8. Dawson P. New definition for relating occlusion to varying conditions of the temporomandibular joint. J Prosthet Dent 1995;74:619-27.

9. Ellis E. Condylar positioning devices for orthognathic surgery: are they necessary? J Oral Maxillofac Surg 1994;52: 536-52.

10. Gill DS, El Maaytah M, Naini FB. Risk factors for post-orthognathic condylar resorption: a review. World J Orthod 2008 Spring;9(1):21-5.

11. Laplanche $O$, Pedeutour $P$, Serre D, Giraudeau A. Condylographie électronique. Cah Prothèse 2002;119:41-57.

12. McMillen LB. Border movements of the human mandible. J Prosthet Dent 1972;27(5):524-32.

13. Posselt U. Studies in the mobility of the human mandible. Acta Odontol Scand 1952;10 (suppl10).

14. Yamauchi K, Takenobu T, Takahashi T. Condylar luxation following bilateral intraoral vertical ramus osteotomy. Oral Surg Oral Med Oral Pathol Oral Radiol Endod 2007 Dec;104(6):747-51. Epub $2007 \mathrm{Jul} 25$.

15. Zak MJ, Dolan E.A, Angelillo JC, McGraw TA. No effect of neuromuscular blockade on the temporomandibular joint position during general anesthesia. Anest Prog 1992;39:212-4. 